\title{
Prevention of Prostate Cancer with Oleanane Synthetic Triterpenoid CDDO-Me in the TRAMP Mouse Model of Prostate Cancer
}

\section{Xiaohua Gao ${ }^{1}$, Dorrah Deeb ${ }^{1}$, Yongbo Liu ${ }^{1}$, Ali S. Arbab ${ }^{2}$, George W. Divine ${ }^{3}$,} Scott A. Dulchavsky ${ }^{1}$ and Subhash C. Gautam ${ }^{1, *}$

1 Department of General Surgery, Henry Ford Health System, Detroit, MI 48150, USA; E-Mails: tgao1@hfhs.org (X.G.); ddeeb1@hfhs.org (D.D.); bliu1@hfhs.org(Y.L.); sdulcha1@hfhs.org (S.A.D.)

2 Department of Diagnostic Radiology, Henry Ford Health System, Detroit, MI 48150, USA; E-Mail: sali1@hfhs.org

3 Department of Public Health Sciences, Henry Ford Health System, Detroit, MI 48150, USA; E-Mail: gdivine1@hfhs.org

* Author to whom correspondence should be addressed; E-Mail: sgautam1@hfhs.org; Tel.: +1-313-874-6998.

Received: 26 May 2011; in revised form: 12 August 2011 / Accepted: 15 August 2011 / Published: 19 August 2011

\begin{abstract}
Cyano-3,12-dioxooleana-1,9(11)-dien-28-oic acid (CDDO), a synthetic analog of oleanolic acid, and its C28 methyl ester derivative (CDDO-Me), have shown potent antitumorigenic activity against a wide range of cancer cell lines, including prostate cancer cells in vitro, and inhibited the development of liver and lung cancer in vivo. In the present study, we examined the efficacy of CDDO-Me in preventing the development and progression of prostate cancer in the transgenic adenocarinoma of the mouse prostate (TRAMP) model. CDDO-Me inhibited the growth of murine TRAMPC-1 prostate cancer cells by inducing apoptosis through the inhibition of antiapoptotic p-Akt, p-mTOR and NF- $\kappa$ B. Early intervention with CDDO-Me $(7.5 \mathrm{mg} / \mathrm{kg})$ initiated at five weeks of age for $20 \mathrm{wk}$ inhibited the progression of the preneoplastic lesions (low-grade PIN and high-grade-PIN) to adenocarcinoma in the dorsolateral prostate (DLP) and ventral prostate (VP) lobes of TRAMP mice. Even delayed administration of CDDO-Me started at $12 \mathrm{wk}$ of age for $12 \mathrm{wk}$ inhibited the development of adenocarcimona of the prostate. Both early and late treatment with CDDO-Me inhibited the metastasis of tumor to the distant organs. Treatment with
\end{abstract}


CDDO-Me inhibited the expression of prosurvival p-Akt and NF- $\mathrm{kB}$ in the prostate and knocking-down Akt in TRAMPC-1 tumor cells sensitized them to CDDO-Me. These findings indicated that Akt is a target for apoptoxicity in TRAMPC-1 cells in vitro and potentially a target of CDDO-Me for inhibition of prostate cancer in vivo.

Keywords: prostate cancer; chemoprevention; CDDO-Me; apoptosis; Akt/mTOR/NF-kB signaling

\section{Introduction}

Carcinoma of the prostate $(\mathrm{CaP})$ is the most commonly diagnosed cancer and second leading cause of cancer-related mortality in men in the United States. Current therapies (radical prostatectomy, local radiotherapy or brachytherapy), while successful for treating localized prostate cancer, are of limited efficacy against metastatic disease [1,2]. Epidemiological studies have shown that high-fat diet and lifestyle are prominent risk factors for prostate cancer [3]. In contrast to the high incidence of prostate cancer in North America and other high-fat diet-consuming countries of the Western Hemisphere, the incidence of prostate cancer is very low in Asian men. Low incidence of prostate cancer among Asian men has been attributed to the consumption of low-fat diet and high intake of dark green vegetables, fruits, and soy products [4]. The cancer-preventing effects of plant-derived foods are due to the presence of polyphenolic phytochemicals with strong antioxidant and anti-inflammatory activity [5]. Indeed, the activity of plant-derived polyphenolic compounds in preventing and/or slowing the progression of prostate cancer has been demonstrated in animal studies and clinical trials [6-8]. Because the incidence of $\mathrm{CaP}$ increases with advancing age and the multistep oncogenic process leading to $\mathrm{CaP}$ is completed over decades, early intervention with natural compounds or their synthetic analogs with proven anti-inflammatory and antioxidant activity represents a promising approach to preventing/delaying the incidence, progression, recurrence, morbidity and mortality associated with the cancer of prostate.

Oleanolic acid and ursolic acid are naturally occurring triterpenoids that have been used in traditional medicine as anti-cancer and anti-inflammatory agents [9-11]. Recent studies have shown that the synthetic derivatives of oleanolic acid such as 2-cyano-3,12-dioxooleana-1,9(11)-dien-28-oic acid (CDDO) and its methyl ester (CDDO-Me) or imidazole (CDDO-Im) derivatives exhibit greater anti-inflammatory and antitumorigenic activity than oleanolic acid itself [12-14]. Synthetic CDDOs have shown potent antiproliferative and antitumorigenic activity against diverse types of tumor cell lines, including leukemia, multiple myeloma, osteosarcoma, breast, brain, prostate and lung cancer cell lines [15-19]. Although the mechanisms of the anticancer effects of CDDOs are not fully understood, cancer cell differentiation, apoptosis and modulation of MAPK (Erk1/2), NF- $\kappa$ B, TGF- $\beta /$ Smad and $\operatorname{PPAR} \gamma$ signaling pathways contribute to the antitumor activity of CDDOs [18,20-24]. CDDOs have also shown chemopreventive activity in animal models of liver, breast and lung cancer [25-27]. We have previously shown that CDDOs inhibit the growth of hormone-sensitive and hormone-refractory prostate cancer cell lines in vitro and in vivo by inducing apoptosis [16,28]. Furthermore, CDDO, the parent compound delayed the progression of prostate cancer in TRAMP mice [29]. 
Our previous in vitro studies showed that CDDO-Me has more potent antiproliferative and antitumorigenic activity against prostate and other cancer cell lines than CDDO [16,30,31]. In the present study, we investigated CDDO-Me for prevention of the development and progression of $\mathrm{CaP}$ in the TRAMP mouse model of prostate cancer. Results showed that treatment with CDDO-Me inhibits the progression of preneoplastic lesions to adenocarcinoma and metastasis of prostate cancer to the distant organs. The tumor inhibitory effect of CDDO-Me was associated with the inhibition of prosurvival p-Akt, NF- $\mathrm{B}$ and $\mathrm{p}-\mathrm{mTOR}$ signaling molecules.

\section{Results}

\subsection{CDDO-Me Inhibits Proliferation and Induces Apoptosis in TRAMPC-1 Prostate Carcinoma Cells}

To test the effect of CDDO-Me on proliferation of prostate cancer cells, $1 \times 10^{4}$ TRAMPC- 1 cells were plated in 96-well microtiter plates for $24 \mathrm{~h}$ and then treated with CDDO-Me for $72 \mathrm{~h}$ at concentrations ranging from 0.625 to $5 \mu \mathrm{M}$. Viability of cultures was determined by MTS assay and percent cytotoxicity (apoptoxicity) calculated from the reduction in viability of cultures. As shown in Figure 1A, significant apoptoxicity was observed in cells treated with CDDO-Me at concentrations of $1.25 \mu \mathrm{M}$ to $5 \mu \mathrm{M}$ (53\% to $60 \%$ ), which increased to nearly $80 \%$ at $10 \mu \mathrm{M}$ (not shown).

To determine whether inhibition of proliferation of TRAMPC-1 cells by CDDO-Me was associated with induction of apoptosis, binding of annexin $\mathrm{V}$ to cells treated with CDDO-Me was measured. For this, TRAMPC-1 cells were treated or not with CDDO-Me (1.25 to $5 \mu \mathrm{M})$ for $24 \mathrm{~h}$ and analyzed for annexin V-FITC binding by flow cytometry. As shown in Figure 1B, the percentage of annexin V-FITC binding TRAMPC-1 cells increased in a dose-dependent manner following treatment with CDDO-Me at $1.25,2.5$ and $5.0 \mu \mathrm{M}(12 \%, 26 \%$ and $63 \%$, respectively). The induction of apoptosis by CDDO-Me was confirmed by the cleavage of native PARP-1 in TRAMPC-1 cells treated with CDDO-Me (Figure 1C). Cells treated with CDDO-Me clearly showed the presence of the $89 \mathrm{kDa}$ cleaved PARP-1 fragment. Together, these results demonstrated that CDDO-Me inhibits proliferation and induces apoptosis in TRAMPC-1 prostate adenocarcinoma cells.

\subsection{CDDO-Me Inhibits Akt (p-Akt) mTOR (p-mTOR) and NF- $\kappa B$ Signaling Proteins in TRAMPC-1 Cells}

Akt, mTOR and NF- $\mathrm{BB}$ are anti-apoptotic (prosurvival) signaling proteins that are constitutively active in a variety of human and animal cancers and provide survival advantage to cancer cells. We investigated whether TRAMPC-1 cells express constitutively active Akt (p-Akt), mTOR (p-mTOR) and NF- $\mathrm{NB}(\mathrm{p}-65)$ and the effect CDDO-Me has on the expression of these signaling proteins. Cell lysates were prepared from TRAMPC- 1 cells treated or not with CDDO-Me (0 to $10 \mu \mathrm{M})$ for $24 \mathrm{~h}$ and analyzed by western blotting. Figure 2 shows that TRAMPC- 1 cells express p-Akt, p-mTOR and p-65 (NF-kB) (lane 1) and CDDO-Me inhibited these activated (phosphorylated) signaling proteins in concentration-related manner. Significant to complete reduction of p-Akt and p-mTOR occurred at concentrations of $1.25 \mu \mathrm{M}$ to $10 \mu \mathrm{M}$ whereas NF- $\kappa \mathrm{B}$ was most affected at 5 to $10 \mu \mathrm{M}$ CDDO-Me. There was no significant change in total protein levels of Akt, mTOR of NF- $\kappa B$ (not shown). These data demonstrated that Akt, mTOR and $\mathrm{NF}-\kappa \mathrm{B}$, all major prosurvival signaling proteins that are critical to the development and progression of prostatic adenocarcinoma are inhibited by CDDO-Me. 
Figure 1. CDDO-Me inhibits growth and induces apoptosis in TRAMPC-1 prostate cancer cells. (A). Effect on cell viability. $1 \times 10^{4}$ TRAMPC- 1 cells were treated with CDDO-Me at concentrations ranging from 0 to $5 \mu \mathrm{M}$ for $72 \mathrm{~h}$ in triplicate in 96-well microtiter plate. Cell viability was measured by MTS assay using CellTiter AQueous assay system from Promega and from decrease in viability cytotoxicity (apoptoxicity) was determined; (B) \& (C). CDDO-Me induces apoptosis in TRAMPC-1 cells. TRAMPC-1 cells were treated with CDDO-Me for $24 \mathrm{~h}$ at concentrations as shown and binding of annexin V-FITC (B) and cleavage of PARP-1 (C) was measured by flow cytometry and western blotting, respectively. ${ }^{*}$ Significantly different from control $(P<0.05)$.

A

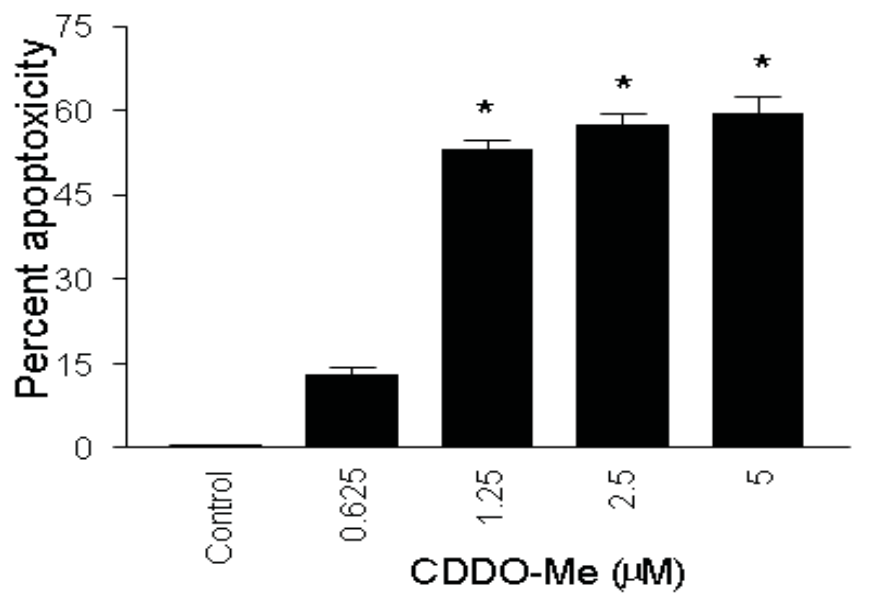

B

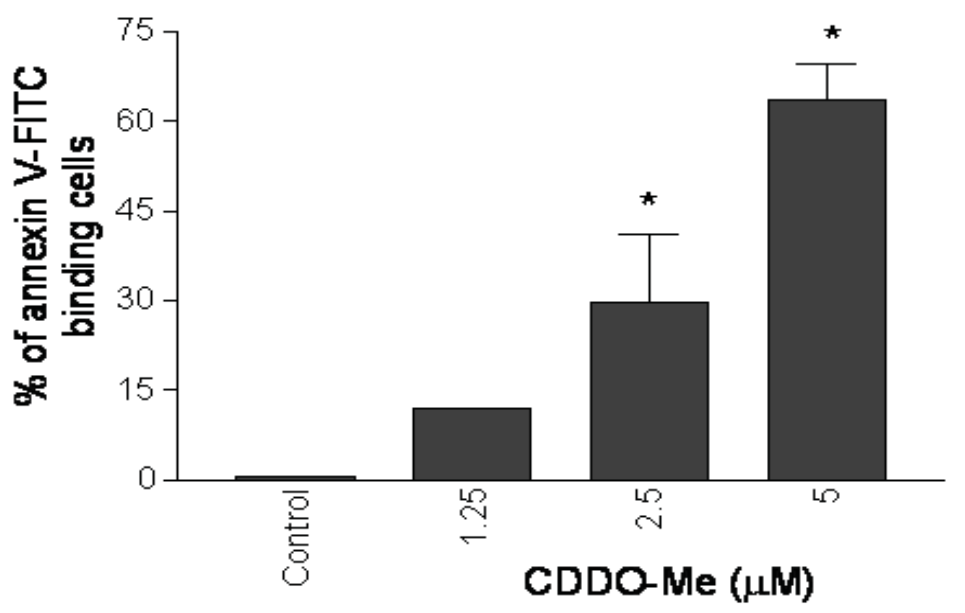

CDDO-Me $(\mu \mathrm{M})$

C

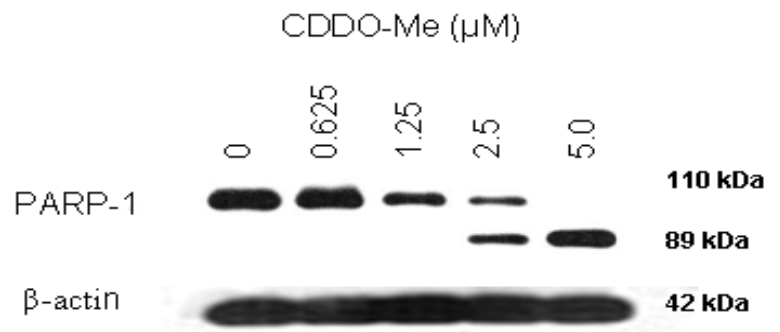


Figure 2. CDDO-Me inhibits antiapoptotic p-Akt, $p-m T O R$ and NF- $\kappa B$ in TRAMPC-1 cells. TRAMPC-1 cells were treated with CDDO-Me $(0-10 \mu \mathrm{M})$ for $24 \mathrm{~h}$ and levels of p-Akt, p-mTOR and NF- $\kappa B(p 65)$ were analyzed by Western blotting.

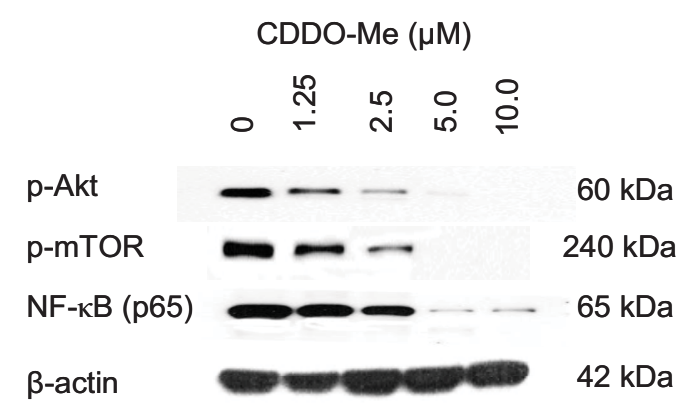

\subsection{CDDO-Me Slows Down the Progression of Prostate Cancer in TRAMP Mice}

Early intervention with CDDO-Me was started at the age of 5 weeks, at which time there is no evidence of neoplastic lesions in the prostate and continued until for 7 (short-term) or 20 weeks (long-term) at a dose of $7.5 \mathrm{mg} / \mathrm{kg} /$ day, 5 days/wk by oral gavage. CDDO-Me was well tolerated without evidence of noticeable toxicity with respect to animal appearance, behavior or change in the body weight. Body weight increased as a function of age at the same rate in both the control and treatment groups and there were no deaths in the control or treatment groups during the entire course of the experiment. In addition, microscopic examination of tissue sections of liver, kidney, lung and small intestine showed no discernable histopathological changes (not shown).

To determine whether CDDO-Me interferes with the development and/or progression of carcinoma of the prostate in TRAMP mice, dorso-lateral (DLP) and ventral prostate (VP) lobes of the TRAMP prostate harvested $7 \mathrm{wk}$ (short-term) or $20 \mathrm{wk}$ (long-term) after treatment with CDDO-Me were evaluated for the incidence and grade of tumor. Visual examination of the abdominal cavity did not reveal unusual enlargement of the seminal vesicles, prostatic lobes or pelvic lymph nodes in control or CDDO-Me treated mice. The majority of the control animals (43\%) showed high-grade PIN (HG-PIN), 21\% showed low-grade PIN (LG-PIN) and 34\% of the animals had well differentiated carcinoma (WD) in the DLP (Figures 3A and a). The distribution of lesions in CDDO-Me treated animals was $46 \%$ LG-PIN, 46\% HG-PIN and 8\% WD. In VP, the distribution of pathologic lesions was very similar in both control and CDDO-Me treated mice (Figures $3 \mathrm{~A}$ and $3 \mathrm{~b}$ ). None of the animals exhibited moderately or poorly differentiated CaP in DLP or VP in either group. Although there was a shift towards normal/non-cancerous pathological grade both in DLP and VP but it did not reach statistical significance $(P>0.05)$.

In mice treated with CDDO-Me for $20 \mathrm{wk}$, tumor growth in seminal vesicles was evident in $71 \%$ of control (10/14) and 14\% of CDDO-Me treated mice (2/14) at necropsy. Histological evaluation of the DLP of control mice showed HG-PIN in $29 \%$ of the mice, well-differentiated adenocarcinoma in $64 \%$ of the animals and one mouse (7\%) exhibited moderately differentiated (MD) adenocarcinoma (Figures 3 B, a). These data indicated that $71 \%$ of the control mice had cancerous lesions in the DLP. In contrast, DLP in $20 \%$ of CDDO-Me treated mice had LG-PIN, 50\% showed HG-PIN and 30\% had WD. These data demonstrated that majority of the CDDO-Me treated animals $(70 \%)$ had noncancerous lesions (LG-PIN and HG-PIN) compared to $71 \%$ of the control mice that showed carcinoma of the prostate $(\mathrm{CaP})$. 
Figure 3. (A). H\&E-stained sections of DLP and VP of a 12 wk old vehicle control or CDDO-Me treated TRAMP mouse. Control and CDDO-Me treated DLP showing HG-PIN and LG-PIN lesions, respectively. Control and CDDO-Me treated VP showing well differentiated carcinoma (MD) and LG-PIN, respectively. Bar graphs show distribution of histologic grades (normal, LG-PIN, HG-PIN, WD, MD or PD carcinoma) in DLP and VP of control and CDDO-Me treated TRAMP mice $(n=14)$; $(\mathbf{B})$. H\&E-stained sections of DLP and VP of a 25 wk old vehicle control or CDDO-Me treated TRAMP mouse. Control and CDDO-Me treated DLP showing well differentiated carcinoma (MD) and HG-PIN lesions, respectively. Control and CDDO-Me treated VP showing MD and LG-PIN, respectively. Bar graphs show distribution of histologic grades (normal, LG-PIN, HG-PIN, WD, MD or PD carcinoma) in DLP and VP of control and CDDO-Me treated mice $(n=14)$.
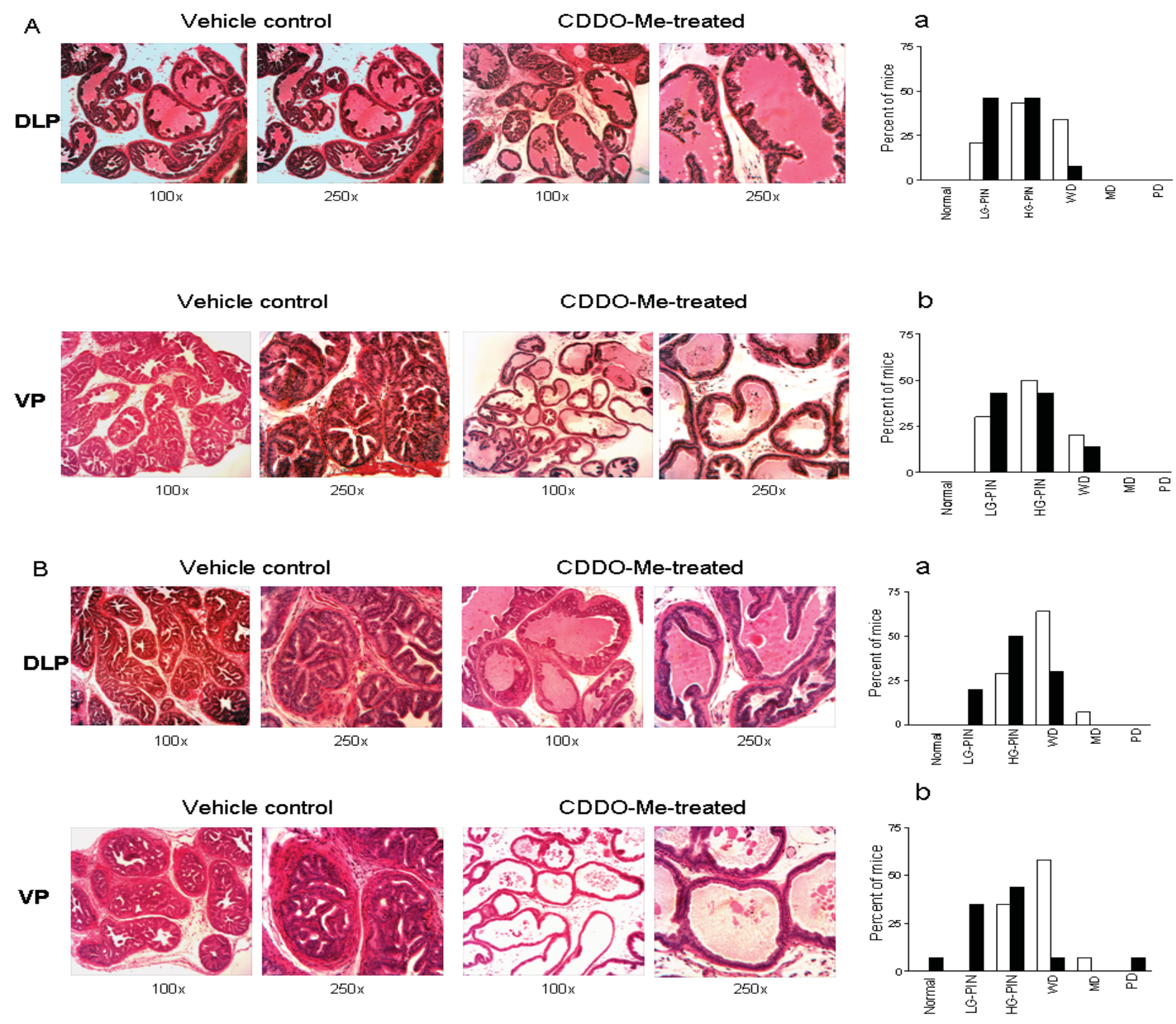

CDDO-Me also inhibited the progression of $\mathrm{CaP}$ in ventral prostate (Figure $3 \mathrm{~B}, \mathrm{~b}$ ). $65 \%$ of the control mice had cancerous lesions ( $\mathrm{WD}=58 \%$; $\mathrm{MD}=7 \%$ ) and 35\% \% had HG-PIN. Among the CDDO-Me treated animals, one mouse showed normal VP (7\%), 79\% had preneoplastic lesions (35\% LG-PIN and $44 \%$ HG-PIN); and 7\% of the mice showed WD and PD each. Both for DLP and VP, CDDO-Me treatment was associated with a shifting in pathologic grade distribution toward normal/noncancerous 
lesions (LG-PIN and HG-PIN, $P<0.01$ ). The overall impact of long-term (20 wk) treatment with CDDO-Me on the incidence and the grade of histologic lesions in the prostate is shown in Table 1. Together, these data showed that although CDDO-Me does not prevent the development of preneoplastic lesions in majority of the animals but it significantly inhibits their progression to $\mathrm{CaP}$.

Table 1. Effect of long-term treatment with CDDO-Me on the incidence and distribution of pathologic lesions in the prostatic lobes.

\begin{tabular}{|l|l|l|l|l|l|l|}
\hline \multirow{2}{*}{$\begin{array}{l}\text { Prostate } \\
\text { lobe }\end{array}$} & \multicolumn{5}{|c|}{ Pre-neoplastic lesions } & \multicolumn{4}{c|}{ Adenocarcinoma } \\
\hline DLP & & & & & & \\
Cormal & & \multicolumn{1}{c|}{ LG-PIN } & \multicolumn{1}{c|}{ HG-PIN } & WD & \\
CDDrol & $0 / 14(0 \%)$ & $0 / 14(0 \%)$ & $4 / 14(29 \%)$ & $9 / 14(64 \%)$ & $1 / 14(7 \%)$ & $0 / 14(0 \%)$ \\
VP & $0 / 14(0 \%)$ & $3 / 14(22 \%)$ & $7 / 14(50 \%)$ & $4 / 14(29 \%)$ & $0 / 14(0 \%)$ & $0 / 14(0 \%)$ \\
Control & & & & & & \\
CDDO-Me & $1 / 14(7 \%)$ & $5 / 14(36 \%)$ & $6 / 14(43 \%)$ & $1 / 14(7 \%)$ & $0 / 14(0 \%)$ & $1 / 14(7 \%)$ \\
\hline
\end{tabular}

DLP: dorsolateral prostate; VP: ventral prostate; LG-PINL: low-grade PIN; HG-PIN: high-grade PIN; WD: well differentiated; MD: moderately differentiated; PD: poorly differentiated

\subsection{Delayed Intervention with CDDO-Me Inhibits CaP Progression in TRAMP Mice}

Whether CDDO-Me inhibits and/or reverses the progression of established neoplastic lesions in DLP and VP was investigated next. Treatment with CDDO-Me was delayed until mice were 12 weeks old since the majority of mice at this age exhibit HG-PIN and well-differentiated adenocarcinoma both in DLP and VP. After treatment with CDDO-Me for $12 \mathrm{wk}$ DLP and VP were examined for histologic tumor grade. As shown in Figure 4A, 71\% of the control mice showed cancerous lesions $(64 \%$ WD and $7 \% \mathrm{MD}$ ) and $29 \%$ had HG-PIN. In contrast, only $21 \%$ of the CDDO-Me treated mice showed WD carcinoma, whereas 79\% had preneoplastic lesions (21\% LG-PIN and 58\% HG-PIN). The distribution of lesions in VP of untreated mice was 65\% cancerous (58\% WD and 7\% MD) and 35\% preneoplastic (HG-PIN). Thirty three percent of CDDO-Me treated mice showed WD carcinoma and $66 \%$ had preneoplastic lesions (34\% LG-PIN and 33\% HG-PIN). These findings demonstrated that delayed treatment with CDDO-Me significantly prevent/ reverse the progression of CaP in both DLP and VP glands in TRAMP mice $(P<0.005$ and $P<0.03$, respectively).

Figure 4. Effect of delayed treatment with CDDO-Me on tumor grade. Bar graphs show distribution of histologic grades (normal, LG-PIN, HG-PIN, WD, MD or PD carcinoma) in DLP (A) and VP (B). Number of animals in each group $=14$.
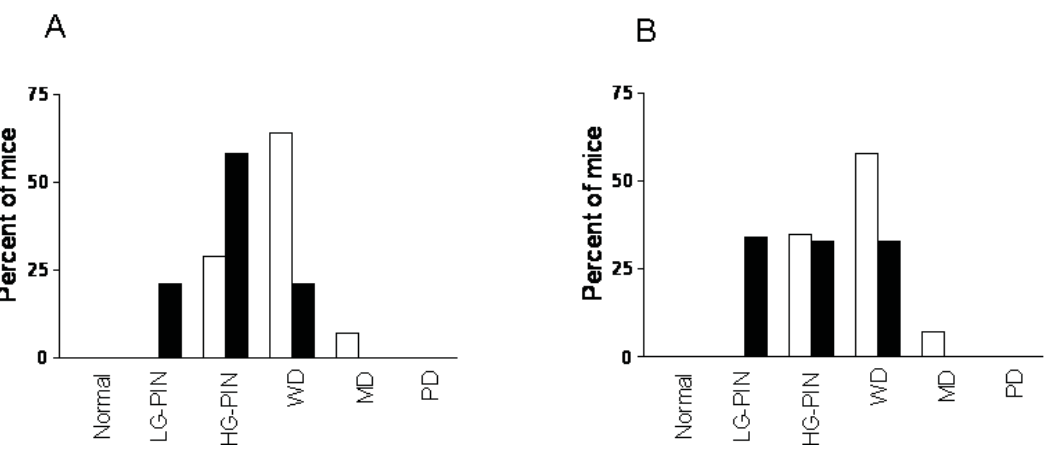


\subsection{CDDO-Me Prevents Metastasis of Prostate Cancer}

At necropsy, pelvic lymph nodes in most of the control mice were slightly enlarged compared to treated mice. No visible lesions were found in lung, liver, kidney in either group. H\&E stained sections of lung, liver, kidney and pelvic lymph nodes were examined for the presence of microscopic metastases. Figure 5A shows the histological appearance of metastatic lesions in the liver (a), lung (b), kidney (c) and pelvic lymph node (d) in a control mouse. As can be seen in Figure 5B, both early (5 wk) and late age (12 wk) intervention with CDDO-Me reduced the incidence of metastasis in lung, liver, kidney and pelvic lymph nodes. The most noticeable anti-metastatic effect of CDDO-Me was observed in the pelvic lymph nodes. Twelve of the 13 control mice $(92 \%)$ showed multiple metastatic foci in the lymph nodes, whereas only 2 of $12(17 \%)$ mice in the early intervention group and 3 of $12(25 \%)$ in the late intervention group had metastatic lesions in the lymph node. Further, the multiplicity of metastases in both treatment groups was markedly reduced compared to the control mice. Overall, these data indicated that CDDO-Me inhibits metastasis of prostate cancer to the distant organs in TRAMP mice.

Figure 5. Effect of CDDO-Me on metastasis of CaP. (A). H\&E-stained sections of liver (a), lung (b), kidney (c) and pelvic lymph node (d) of $25 \mathrm{wk}$ old control TRAMP mice showing metastatic lesions (arrows) $\times 250$ magnification; (B). Bar graph showing the incidence of metastasis in different organs in control and CDDO-Me treated TRAMP mice. The number of CDDO-Me treated mice with lymph node metastasis was significantly reduced $(P<0.01)$.

A

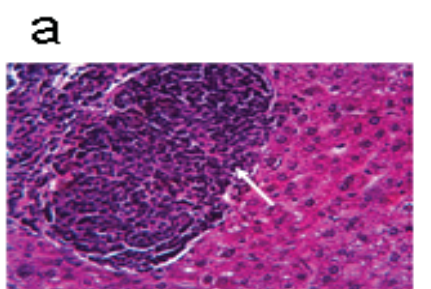

b
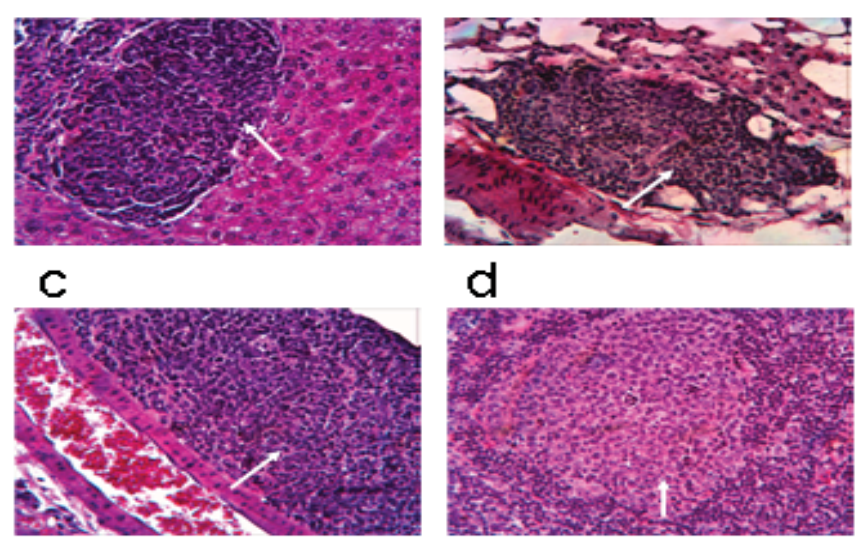

d

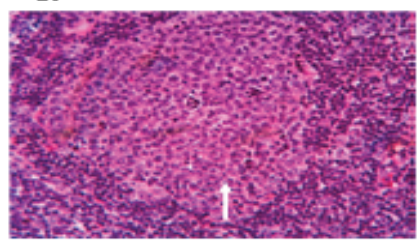

B

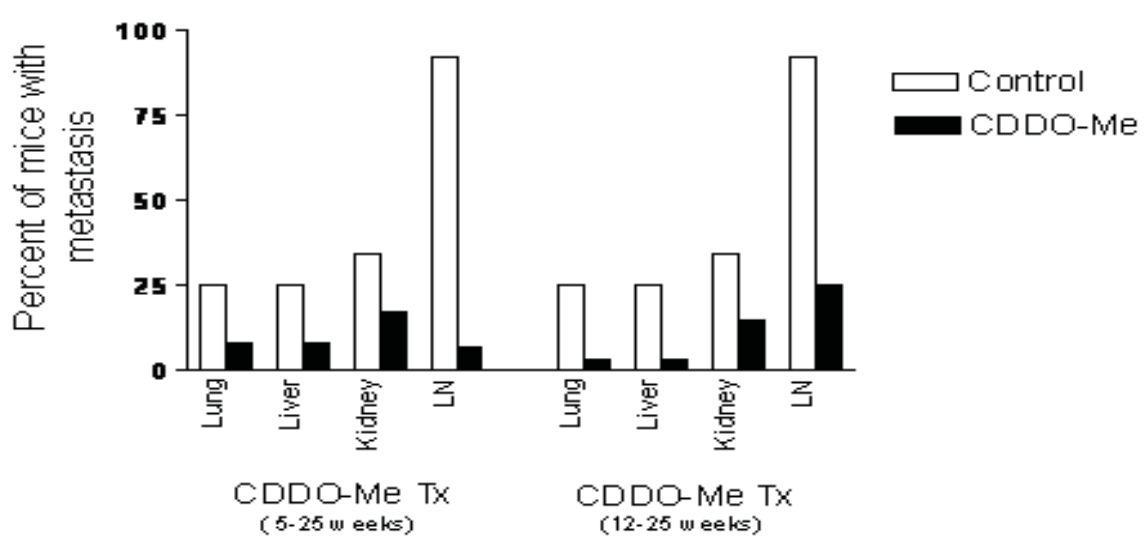




\subsection{CDDO-Me Reduces Levels of p-Akt and NF- $k B$ (P65) in Dorsolateral Prostate}

Akt and NF- $\mathrm{KB}$ are major antiapoptotic molecules that confer survival advantage and resistance to anticancer therapies in cancer cells. Here we investigated whether p-Akt and NF- $\mathrm{BB}$ expression was altered in the DLP of mice treated with CDDO-Me for $20 \mathrm{wk}$. Figure 6A compares the levels of p-Akt and NF- $\mathrm{KB}$ in the DLP of control and CDDO-Me treated mice (seven each). Blot clearly shows an overall inhibition of p-Akt (40\% reduction) and NF- $\kappa \mathrm{B}(25 \%$ reduction) in the DLP of CDDO-Me treated mice compared to control mice. The levels of basal Akt or p65 were not affected by CDDO-Me (not shown).

Figure 6. CDDO-Me inhibits p-Akt and NF- $\mathrm{kB}$ (p65) in DLP and knocking-down sensitizes prostate cancer cells to CDDO-Me. (A). DLP lysates prepared from control and CDDO-Me treated TRAMP mice were fractionated on polyacrylamide gel and $\mathrm{p}$-Akt and NF- $\kappa \mathrm{B}$ were detected by immunoblotting; (B). TRAMPC-1 cells were transfected with double stranded siRNA-Akt as described in Materials and Methods and sensitivity of transfected cells to CDDO-Me was measured in MTS assay. * Significantly different from control $(P<0.01)$.

A

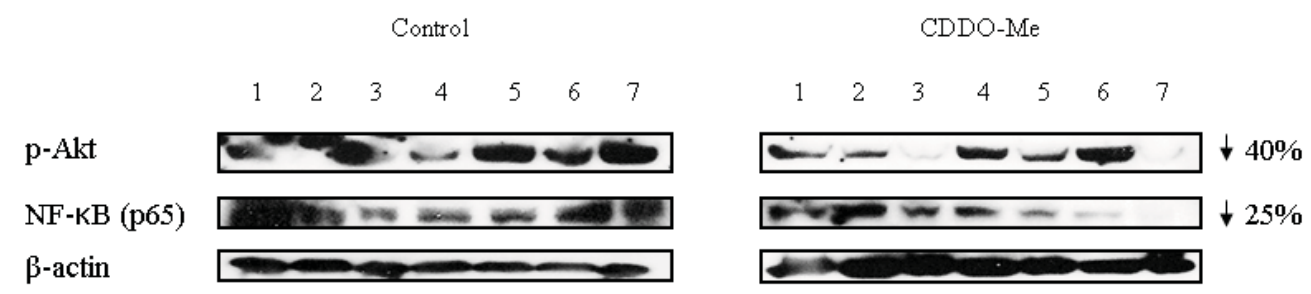

B

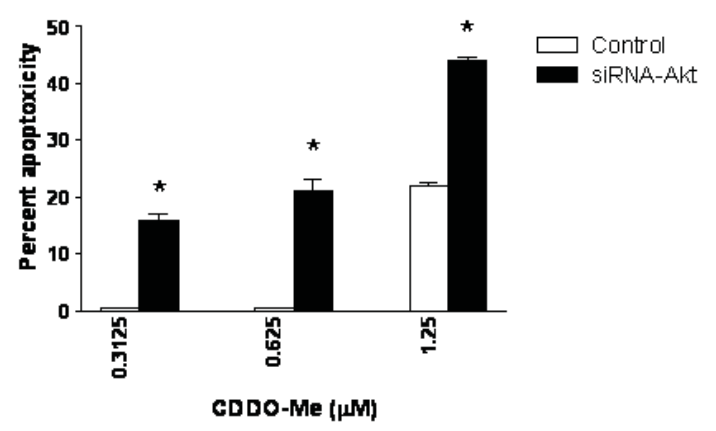

Since Akt plays a central role in the regulation of both NF- $\mathrm{B}$ and mTOR we evaluated the significance of Akt inhibition in killing of prostate cancer cells by CDDO-Me. For this, Akt was knocked-down in TRAMPC-1 tumor cells with siRNA-Akt and their response to low concentrations of CDDO-Me was measured in MTS assay. Figure 6B shows that the sensitivity of TRAMPC-1 cells to low concentrations of CDDO-Me is increased following transfection with siRNA-Akt (CDDO-Me treated cells $=16 \%, 21 \%$ and $44 \%$ cytotoxicity; control cells $=0 \%, 2 \%$ and $22 \%$ cytotoxicity at 0.325 , 0.625 and $1.25 \mu \mathrm{M}$ CDDO-Me, respectively). Minimally, increase in the sensitivity of transfected cells suggests that Akt is a good target for apoptoxicity in TRAMPC-1 cells in vitro, however, considering 
that it is also decreased in the DLP of animals treated with CDDO-Me indicates that Akt is potentially a target of CDDO-Me in prevention of prostate cancer in TRAMP mice.

\subsection{Discussion}

The present study is an extension of our previous study in which we demonstrated the efficacy of CDDO, the parent synthetic analog of oleanolic acid, in preventing the progression of preneoplastic lesions of prostate cancer in TRAMP mice [29]. Our in vitro studies have shown that CDDO and its methyl ester (CDDO-Me) or imidazole (CDDO-Im) potently inhibit cell proliferation and induce apoptosis in human and mouse prostate cancer cell lines [16,28,30,30]. These studies also demonstrated that CDDO was weaker than CDDO-Me or CDDO-Im in inhibiting the growth of prostate and other cancer cells in culture [16,30,31]. Since our recent study showed partial inhibition of the progression of preneoplastic lesions to $\mathrm{CaP}$ in TRAMP mice by CDDO [29], we proceeded to investigate the efficacy of CDDO-Me for preventing the development and progression of $\mathrm{CaP}$ in TRAMP mice. Oral treatment with CDDO-Me for 20 weeks was tolerated well, without toxic side effects, a prerequisite for considering new agents for human trial. Short-term early intervention (7 wk) with CDDO-Me had weak but measurable suppressive effect on the development and progression of preneoplastic LG-PIN and HG-PIN lesions in TRAMP mice. On the other hand, long-term early intervention (20 wk) with CDDO-Me showed potent inhibition of the progression of preneoplastic lesions in DLP and VP to invasive adenocarcinoma. Treatment with CDDO-Me for 20 weeks resulted in a significant shift in pathologic grade distribution toward normal/noncancerous lesions compared to the predominance of cancerous lesions in control animals. Thus, although CDDO-Me did not prevent the development of preneoplastic lesions in majority of the animals it significantly prevented their progression to the cancerous lesions. These findings are in agreement with the previously reported chemopreventive effects of CDDO analogs in aflatoxin-induced hepatic carcinogenesis and vinyl carbamate-induced lung cancer [25,27] and of herbal products such as sulforaphane, green tea polyphenols, garlic constituents and genistein on prostate tumorigenesis in TRAMP mice [6,7,32,33]. The present study showed that despite of the differences in the growth inhibitory effect of CDDO and CDDO-Me on prostate cancer cell lines in vitro, the chemopreventive efficacy of these synthetic oleanane analogs for $\mathrm{CaP}$ in the TRAMP mice is comparable. This may be due to the long-term treatment of mice with the two agents versus short term exposure of tumor cells in culture systems. Our results showed that even delayed intervention with CDDO-Me in animals with established $\mathrm{CaP}$ also prevented/reversed the progression of prostate cancer, suggesting therapeutic potential of CDDO-Me for prostate cancer.

Although we did not explore the mechanism of the anti-metastatic activity of CDDO-Me in this study, both early and delayed intervention with CDDO-Me reduced the incidence of metastasis of prostate cancer to liver, lung, kidney and pelvic lymph nodes. CDDO-Me was most effective in preventing metastasis of $\mathrm{CaP}$ to the pelvic lymph nodes. There are multiple potential targets for the antimetastatic effects of CDDO-Me, including cell adhesion molecules (E-cadherin), angiogenic growth factors and matrix metalloproteinases that remain to be explored. Because bone metastasis in TRAMP mice occurs after 30 wk of age and beyond we were not able to evaluate it in this study. It is 
imperative however to determine the antimetastatic activity of CDDO-Me for bone, since prostate cancer in men predominantly metastasizes to the bone.

In vitro studies showed that inhibition of proliferation and induction of apoptosis in TRAMPC-1 cancer cells by CDDO-Me was associated with inhibition of $\mathrm{p}-\mathrm{Akt}, \mathrm{p}-\mathrm{mTOR}$ and NF-kB. These signaling proteins play critical role in tumor development, growth and spread by regulating cell proliferation, apoptosis, inflammation, angiogenesis, and metastasis. Activated p-Akt promotes cell survival by inactivating downstream substrates such as $\mathrm{p}$-Bad, procaspase-9, and Forkhead transcription factors [34,35]. Antiapoptotic NF- $\mathrm{kB}$ controls the expression of genes involved in inflammation, proliferation, oncogenesis, angiogenesis, and apoptosis [36] whereas mTOR regulates cell growth, survival, ribogenesis and translation [37]. These signaling proteins are constitutively active in the DLP of the TRAMP mice and are potential targets of tumor inhibition by natural polyphenolic compounds [38]. We investigated whether CDDO-Me alters the expression of $\mathrm{p}-\mathrm{Akt}$ or NF- $\mathrm{kB}$ (p65) in DLP in TRAMP mice. Indeed, treatment with CDDO-Me reduced the levels of both p-Akt and NF- $\mathrm{B}$ (p65) in the DLP compared to control mice. Furthermore, knocking-down Akt increased the sensitivity of tumor cells to CDDO-Me, indicating that inhibition of these prosurvival (antiapoptotic) proteins is part of the mechanism by which CDDO-Me inhibits prostate tumorigenesis. This conclusion however needs more direct studies to prove the relevance of these molecules in preventing the development and progression of prostate cancer since inhibition of these signaling proteins could be independent and unrelated to the mechanisms of CDDO-Me.

In summary, we have shown the efficacy of CDDO-Me in inhibiting the progression of prostate cancer and its metastasis to the distant sites in the TRAMP mouse model of prostate tumorigenesis. Furthermore, Akt and its downstream targets such as NF- $\kappa \mathrm{B}$ and mTOR appear to mediate the chemopreventive activity of CDDO-Me.

\section{Experimental}

\subsection{Reagents and Antibodies}

CDDO-Me was obtained from the Developmental Therapeutics Program, National Cancer Institute (Bethesda, MD, USA) through the Rapid Access to Intervention Development Program. Anti- p-Akt $\left(\operatorname{ser}^{473}\right.$ ) antibody was from Cell Signaling Technology (Danvers, MA, USA); anti-NF-kB (p65) and anti-p-mTOR antibody was from Santa Cruz Biotechnology, Inc. (Santa Cruz, CA, USA). 96 AQueous One Solution Proliferation Assay System was from Promega (Madison, WI, USA). 100 mM stock solution of CDDO-Me was prepared in DMSO and all test concentrations were prepared by diluting the stock solution in appropriate medium.

\subsection{Cell Line}

TRAMPC-1 prostate cancer cell line derived from a primary tumor in the prostate of a TRAMP mouse was obtained from American Type Culture Collection (ATCC, Rockville, MD, USA). Cells were grown in DMEM supplemented with $4 \mathrm{mM}$ L-glutamine, $0.005 \mathrm{mg} / \mathrm{mL}$ bovine insulin, $10 \mathrm{nM}$ dehydroisoandrosterone, $5 \%$ fetal bovine serum, and $5 \% \mathrm{Nu}-\mathrm{Serum} \mathrm{IV}$. Cells were cultured at $37{ }^{\circ} \mathrm{C}$ in 
a humidified atmosphere consisting of $5 \% \mathrm{CO} 2$ and $95 \%$ air. Cultures were maintained by subculturing cells twice a week.

\subsection{Mice}

TRAMP mice were bred by the Jackson Laboratories (Bar Harbor, ME, USA) through their Speed Expansion Service involving in vitro fertilization of C57BL/6 females with C57BL/6-Tg (TRAMP) $8247 \mathrm{NG} / \mathrm{J}$ males. Mice were genotyped for the transgene (Tag) and delivered to us when 4 wk old. Mice were maintained in temperature-controlled room $\left(68-72{ }^{\circ} \mathrm{F}\right)$ with a $12 \mathrm{~h} \mathrm{light/dark}$ cycle and provided semi-purified AIN-76A mouse chow and water ad libitum. Mice were acclimated for one week before starting the experiment. All animal treatments were according to the protocol approved by the Institutional Animal Care and Use Committee (IACUC).

\subsection{Treatment Protocol}

Seventy five weeks old male TRAMP mice were weighed and randomized into vehicle control $(n=28)$, early intervention $(n=28)$ and delayed treatment $(n=14)$ groups. Mice in the vehicle control group were administered $0.1 \mathrm{~mL}$ of vehicle consisting of cremophor-EL:DMSO:PBS (1:1:8), 5 days a week by oral gavage for 7 or 20 weeks. In the early intervention group, mice were administered CDDO-Me at a dose of $7.5 \mathrm{mg} / \mathrm{kg}$ in $0.1 \mathrm{~mL}$ of vehicle, 5 days a week for 7 weeks $(\mathrm{n}=14)$ or 20 weeks $(n=14)$. In the delayed treatment group to model the effect on already established tumors, animals were treated with CDDO-Me beginning at the age of 12 weeks through 25 weeks of age. We chose to treat animals with a dose of $7.5 \mathrm{mg} / \mathrm{kg}$ CDDO-Me since 5 to $7.5 \mathrm{mg} / \mathrm{kg}$ CDDO or CDDO-Me was found to inhibit tumor progression in TRAMP and xenograft models of CaP $(28,29)$. Body weight of control and CDDO-Me-treated mice was recorded each week and mice were observed for treatment related stress, such as water and food withdrawal, unusual posture, ruffled fur or listlessness. At the age of 12 weeks (short-term treatment) and 25 weeks (long-term treatment), 14 mice from each group were sacrificed $24 \mathrm{~h}$ after the last administration of vehicle or CDDO-Me. After opening the abdomen, mice were visually observed for the presence of tumor mass, enlargement of seminal vesicles, prostate lobes and pelvic lymph nodes. Dorso-lateral (DLP) and ventral (VP) prostatic lobes, liver, lung, kidney and pelvic lymph nodes were harvested. Tissue samples were processed for histological and biochemical analyses.

\subsection{Pathological Grading of Lesions and Detection of Metastases}

The pathologic grading of prostate cancer was according to the grading system for TRAMP described by Greenberg and colleagues $[39,40]$. Following this grading system, prostate lesions in the DLP and ventral prostate lobes were histologically graded as normal (ducts lined with single layer of secretory epithelial cells surrounded by 2-3 cell layers of fibromuscular stroma; low-grade PIN (epithelial cells with variably elongated nuclei with condensed chromatin); high-grade PIN (epithelial stratification and tufting, presence of micropapillary and cribiform structures); well differentiated (WD) carcinoma (epithelial cells invading fibromuscular stroma) and moderately (MD) to poorly differentiated (PD) adenocarcinoma of the prostate (sheets of neoplastic cells with little or no glandular 
structures). Ten randomly selected microscopic fields on H\&E stained sections of the DLP and VP were scored for the incidence and the pathologic grade of the prostate cancer in control and CDDO-Me-treated TRAMP mice. For the incidence of metastasis (percentage of mice with metastatic lesions), H\&E stained sections of liver, lung, kidney and pelvic lymph nodes were evaluated microscopically.

\subsection{MTS Assay}

Cells $\left(1 \times 10^{4}\right)$ were seeded into each well of a 96-well plate in $100 \mu \mathrm{L}$ of tissue culture medium. After $24 \mathrm{~h}$ incubation to allow cells to adhere, cultures were treated with CDDO-Me at concentrations of $0.625 \mu \mathrm{M}$ to $5 \mu \mathrm{M}$ for $72 \mathrm{~h}$. Cell viability was then determined by the colorimetric MTS assay using CellTiter 96 AQueous One Solution Proliferation Assay System.

\subsection{Western Blotting}

TRAMPC-1 cell and DLP tissue lysates were prepared in lysis buffer (1\% Triton-X 100 (v/v), $10 \mathrm{mM}$ Tris- $\mathrm{HCl}$ (pH 7.5), $5 \mathrm{mM}$ EDTA, $150 \mathrm{mM} \mathrm{NaCl}, 10 \%$ glycerol, $2 \mathrm{mM}$ sodium vanadate, $5 \mu \mathrm{g} / \mathrm{mL}$ leupeptin, $1 \mu \mathrm{g} / \mathrm{mL}$ aprotinin, $1 \mu \mathrm{g} / \mathrm{mL}$ pepstatinin, and $10 \mu \mathrm{g} / \mathrm{mL} 4-2$-aminoethylbenzenesulfinyl fluoride). Lysates were clarified by centrifugation at $14,000 \mathrm{x} g$ for $10 \mathrm{~min}$ at $4{ }^{\circ} \mathrm{C}$, and protein concentrations were determined by Bradford assay. Samples $(50 \mu \mathrm{g})$ were boiled in an equal volume of sample buffer (20\% glycerol, 4\% SDS, 0.2\% Bromophenol Blue, $125 \mathrm{mM}$ Tris- $\mathrm{HCl}(\mathrm{pH} 7.5)$, and $640 \mathrm{mM}$ 2-mercaptoethanol) and separated on pre-casted Tris-glycine polyacrylamide gels using the XCell Surelock ${ }^{\mathrm{TM}}$ Mini-Cell, in Tris-Glycine SDS running buffer, all from Novex (Invitrogen, Carlsbad, CA, USA). Proteins resolved on the gels were transferred to nitrocellulose membranes. Membranes were blocked with 5\% milk in $10 \mathrm{mM}$ Tris- $\mathrm{HCl}(\mathrm{pH} \mathrm{8.0)}, 150 \mathrm{mM} \mathrm{NaCl}$ with $0.05 \%$ Tween 20 (TPBS) and probed using protein specific antibodies to p-Akt $\left(\operatorname{ser}^{473}\right), \mathrm{NF}-\kappa \mathrm{B}(\mathrm{p} 65)$, p-mTOR or $\beta$-actin (loading control) and HRP-conjugated secondary antibody. Immune complexes were visualized using chemiluminescence reagent from Thermo Fisher Scientific (Rockford, IL, USA). Protein bands were imaged and band densities analyzed using the NIH/Scion image analysis software.

\subsection{Histology}

Tissue specimens of prostate gland, liver, lung, kidney, small intestine and pelvic lymph nodes were fixed in 10\% neutral buffered formalin for $48 \mathrm{~h}$ and then embedded in paraffin. Five micrometer thick sections were cut and stained with $\mathrm{H} \& \mathrm{E}$ for routine histology. Histologic interpretations of tumor grade and tumor metastasis were made in a blinded fashion.

\section{9. siRNA Transfection}

For silencing of Akt, TRAMPC-1 cells were transfected with double stranded siRNA-Akt using SignalSilence siRNA kit from Cell Signaling Technology (Beverly, MA, USA). Briefly, $10^{6}$ cancer cells were plated in $60 \mathrm{~mm}$ Petri dish for $24 \mathrm{~h}$ and treated with $3 \mathrm{~mL}$ of transfection medium containing $20 \mu \mathrm{g}$ LipofectAMINE and $100 \mathrm{nM}$ siRNA for $24 \mathrm{~h}$. Gene silencing in transfected cells was confirmed by western blotting. 


\subsection{Statistical Analysis}

Most outcomes for in vitro experiments were compared by Student's t-test. Wilcoxon rank sum tests were used for histologic grade which was scored from 1(normal) to 6 (poorly differentiated) as described above.

\section{Conclusions}

This study has demonstrated the ability of CDDO-Me, an oleanane synthetic triterpenoid in preventing the progression of preneoplastic lesions to the adenocarcinoma of the prostate in TRAMP mouse model. The growth inhibitory and apoptosis-inducing activity of CDDO-Me contributed to the chemoprevention of prostate tumorigenesis. Furthermore, prosurvival Akt/NF- $\mathrm{kB} / \mathrm{mTOR}$ signaling pathway appears to mediate the chemopreventive activity of CDDO-Me. Thus, our mechanism based preclinical study provides support for clinical trial of CDDO-Me for chemoprevention of prostate cancer.

\section{Acknowledgements}

This work was supported by the National Institutes of Health grant 5 R01 CA130948 to S.C.G.

\section{References}

1. Garnick, M.B. Hormonal therapy in the management of prostate cancer: From Higgins to the present. Urology 1997, 49, 5-15.

2. Hanks, GE. Long-term control of prostate cancer with radiation. Urol. Clin. North Amer. 1996, 23, 605-616.

3. Fleshner, N.E.; Bagnell, P.S.; Klotz, L.H.; Venkateswaran, V. Dietary fat and prostate cancer. J. Urol. 2004, 171, S19-24.

4. Block, G.; Patterson, B.; Subar, A. Fruit, vegetables, and cancer prevention: A review of the epidemiological evidence. Nutr. Cancer 1992, 18, 1-29,

5. Kuo, S.M. Dietary flavonoids and cancer prevention: Evidence and potential mechanism. Crit. Rev. Oncogenesis 1997, 8, 47-69.

6. Gupta, S.; Hastak, K.; Ahmad, N.; Lewin, J.S.; Mukhtar, H. Inhibition of prostate carcinogenesis in TRAMP mice by oral infusion of green tea polyphenols. Proc. Natl. Acad. Sci. USA 2001, 98, 10350-10355.

7. Singh, S.V.; Warin, R.; Xiao, D.; Powolny, A.A.; Stan, S.D.; Arlotti, J.A.; Zeng, Y.; Hahm, E-R.; Marynowski, S.W.; Bommareddy, A.; Desai, D.; Amin, S.; Parise, R.A.; Beumer, J.H.; Chambers, W.H. Sulforaphane inhibits prostate carcinogenesis and pulmonary metastasis in TRAMP mice in association with increased cytotoxicity of natural killer cells. Cancer Res. 2010, 69, 2117-2125.

8. Bettuzzi S.; Brausi M.; Rizzi F.; Castagnetti, G.; Peracchia, G.; Corti, A. Chemoprevention of human prostate cancer by oral administration of green tea catechins in volunteers with high-grade prostate intraepithelial neoplasia: A preliminary report from a one-year proof-of-principle study. Cancer Res. 2006, 66, 1234-1240. 
9. Huang, M.T.; Ho, C.T.; Wang, Z.Y.; Ferraro, T.; Lou, Y.R.; Stauber, K.; Ma, W.; Georgiadis, C.; Laskin, J.D.; Conney, A.H. Inhibition of skin tumorigenesis by rosemary and its constituents carnosol and ursolic acid. Cancer Res. 1994, 54, 701-707.

10. Nishino, H.; Nishino, A.; Takayasu, J.; Hasegawa, T.; Iwashima, A.; Hirabayashi, K.; Iwata, S.; Shibata, S. Inhibition of the tumor-promoting action of 12-O-tetradecanoylphorbol-13-accetate by some oleanane-type triterpenoid compounds. Cancer Res. 1988, 48, 5210-5215.

11. Ryu, S.Y.; Oak, M.H.; Yoon, S.K.; Cho, D.I.; Yoo, G.S.; Kim, T.S.; Kim, K.M. Anti-allergic and anti-inflammatory triterpenes from the herb of Prunella vulgaris. Planta Med. 2000, 66, 358-360.

12. Honda, T.; Rounds, B.V.; Gribble, G.W.; Suh, N.; Wang, Y.; Sporn, M.B. Design and synthesis of 2-cyano-3, 12-dioxoolean-1, 9-dien-28-oic acid, a novel and highly active inhibitor of nitric oxide production in mouse macrophages. Biorg. Med. Chem. Lett. 1998, 8, 2711- 2714.

13. Suh, N.; Honda, T.; Finlay, H.J.; Barchowsky, A.; Williams, C.; Benoit, N.E.; Xie, Q.W.; Nathan, C.; Gribble, G.W.; Sporn, M.B. Triterpenoids suppress inducible nitric oxide synthase (iNOS) and inducible cyclooxigenase (COX-2) in mouse macrophages. Cancer Res. 1998, 58, 717-723.

14. Honda, T.; Rounds, B.V.; Bore, L.; Favaloro, F.G.; Gibble, G.W.; Suh, N.; Wang, Y.; Sporn, M.B. Novel synthetic oleanane triterpenoids: A series of highly active inhibitors of nitric oxide production in mouse macrophages. Bioorg. Chem. Lett. 1999, 9, 3429-3434.

15. Gao, X.; Deeb, D.; Jiang, H.; Liu, Y.; Dulchavsky, S.; Gautam S. Synthetic triterpenoids inhibit growth and induce apoptosis in human glioblastoma and neuroblastoma cells through inhibition of prosurvival Akt, NF- $\mathrm{KB}$ and Notch1 signaling. J. Neurooncol. 2007, 84, 147-157.

16. Deeb, D.; Gao, X.; Dulchavsky, S.A.; Gautam, S.C. CDDO-Me induces apoptosis and inhibits Akt, mTOR and NF-kB in prostate cancer cells. Anticancer Res. 2007, 27, 3035-3044.

17. Konopleva, M.; Tsao, T.; Ruvolo, P.; Stiouf, I.; Estrov, Z.; Leysath, C.E.; Zhao, S.; Harris, D.; Chang, S.; Jackson, C.E.; Munsell, M.; Suh, N.; Gribble, G.; Honda, T.; May, W.S.; Sporn, M.B.; Andreeff, M. Novel triterpenoid CDDO-Me is a potent inducer of apoptosis and differentiation in acute myelogenous leukemia. Blood 2002, 99, 326-335.

18. Shishodia, S.; Sethi, G.; Konopleva, M.; Andreeff, M.; Aggarwal, B.B. A synthetic triterpenoid, CDDO-Me, inhibits I kappa B alpha kinase and enhances apoptosis induced by TNF and chemotherapeutic agents through down-regulation of expression of nuclear factor kappa B-regulated gene products in human leukemic cells. Clin. Cancer Res. 2006, 12, 1828-1838.

19. Ikeda, T.; Sporn, M.; Honda, T.; Gribble, G.W.; Kufe, D. The novel triterpenoid CDDO and its derivatives induce apoptosis by disruption of intracellular redox balance. Cancer Res. 2003, 63, 5551-5558.

20. Konopleva, M.; Tsao, T.; Estrov, Z.; Lee, R.M.; Wang, R.Y.; Jackson, C.E.; McQueen, T.; Monaco, G.; Munsell, M.; Belmont, J.; Kantarjian, H.; Sporn, M.B.; Andreeff, M. The synthetic triterpenoid 2-cyano-3, 12-dioxooleana-1, 9-dien-28-oic acid induces caspase-dependent and independent apoptosis in acute myelogenous leukemia. Cancer Res. 2004, 64, 7927-7935.

21. Ito, Y.; Pandey, P.; Sporn, M.B.; Datta, R.; Kharbanda, S.; Kufe. D. The novel triterpenoid CDDO induces apoptosis and differentiation of human osteosarcoma cells by a caspase- 8 dependent mechanism. Mol. Pharmacol. 2001, 59, 1094-1099. 
22. Konopleva, M.; Contractor, R.; Kurinna, S.M.; Chen, W.; Andreeff, M.; Ruvolo, P.P. The novel triterpenoid CDDO-Me suppresses MAPK pathways and promotes p38 activation in acute myeloid leukemia cells. Leukemia 2005, 19, 1350-1354.

23. Suh, N.; Roberts, A.B.; Birkey Reffey, S.; Miyazono, K.; Itoh, S.; ten Dijke, P.; Heiss, E.H.; Place, A.E.; Risingsong, R.; Williams, C.R.; Honda, T.; Gribble, G.W.; Sporn, M.B. Synthetic triterpenoids enhance transforming growth factor beta/Smad signaling. Cancer Res. 2003, 63, 1371-1376.

24. Chintharlapalli, S.; Papineni, S.; Konopleva, M.; Andreef, M.; Samudio, I.; Safe, S. 2-Cyano3,12-dioxoolean-1,9-dien-28-oic acid and related compounds inhibit growth of colon cancer cells through peroxisome proliferator-activated receptor gamma-dependent and -independent pathways. Mol. Pharmacol. 2005, 68, 119-128.

25. Yates, M.S.; Kwak, M.K.; Egner, P.A.; Groopman, J.D.; Bodreddigari, S.; Sutter, T.R.; Baumgartner, K.J.; Roebuck, B.D.; Liby, K.T.; Yore, M.M.; Honda, T.; Gribble, G.W.; Sporn, M.B.; Kensler, T.W. Potent protection against aflatoxin-induced tumorigenesis through induction of Nrf2-regulated pathways by the triterpenoid 1-[2-cyano-3-,12-dioxooleana-1,9(11)-dien-28oyl]imidazole. Cancer Res. 2006, 66, 2488-2494.

26. Xiaoyang, L.; Konopleva, M.; Zeng, Z.; Ruvolo, V.; Stephens, L.C.; Schober, W.; McQueen, T.; Dietrich, M.; Madden, T.L.; Andreeff, M. The novel triterpenoid C-28 methyl ester of 2-cyano-3, 12-dioxoolen-1, 9-dien-28-oic acid inhibits metastatic murine breast tumor growth through inactivation of STAT3 signaling. Cancer Res. 2007, 67, 4210-4218.

27. Liby, K.; Royce, D.B.; Williams, C.R.; Risingsong, R.; Yore, M.M.; Honda, T.; Gribble, G.W.; Dmitrovsky, E.; Sporn, T.A.; Sporan MB. The synthetic triterpenoid CDDO-methyl ester and CDDO-amide prevent lung cancer induced by vinyl carbomate in A/J mice. Cancer Res. 2007, 67, 2414-2419.

28. Deeb, D.; Gao, X.; Jiang, H.; Dulchavsky, S.A.; Gautam, S.C. Oleanane triterpenoid CDDO-Me inhibits growth and induces apoptosis in prostate cancer cells by independently targeting pro-survival Akt and mTOR. Prostate 2009, 69, 851-860.

29. Deeb, D.; Gao, X.; Liu, Bo.; Jiang, D.; Divine, G.; Arbab, S.A.; Dulchavsky, S.A.; Gautam, S.C. Synthetic triterpenoid CDDO prevents the progression and metastasis of prostate cancer in TRAMP mice by inhibiting survival signaling. Carcinogenesis 2011, doi:10.1093/carcin/bgr030.

30. Deeb, D.; Gao, X.; Dulchavsky, S.A.; Gautam, S.C. CDDO-Me inhibits proliferation, induces

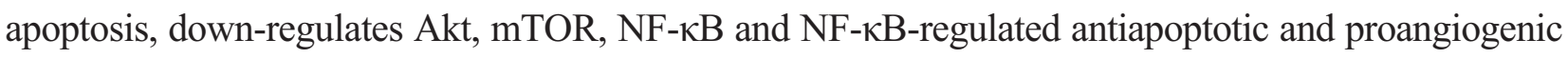
proteins in TRAMP prostate cancer cells. J. Exp. Therapeut. Oncol. 2008, 7, 31-39.

31. Gao, X.; Deeb, D.; Jiang, H.; Liu, Y.; Arbab, Ali S. Dulchavsky, S.; Gautam S.C. Synthetic triterpenoids inhibit growth, induce apoptosis and suppress pro-survival Akt, mTOR and NF-кB signaling proteins in colorectal cancer cells. Anticancer Res. 2010, 30, 785-792.

32. Singh, S.V.; Powolny, A.A.; Stan, S.D.; Xiao, D.; Arlotti, J.A.; Warin, R.; Hahm, E-R.; Marynowki, S.W.; Bommareddy, A.; Potter, D.M.; Dhir, R. Garlic constituent diallyl trisulfide prevents development of poorly differentiated prostate cancer and pulmonary metastasis multiplicity in TRAMP mice. Cancer Res. 2008, 68, 9503-9511.

33. Mentor-Marcel, R.; Lamartiniere, C.A.; Eltoum, I.E.; Greenberg, N.M.; Elgavish, A. Genistein in the diet reduces the incidence of poorly differentiated prostatic adenocarcinoma in transgenic mice (TRAMP). Cancer Res. 2001, 61, 6777-6782. 
34. Datta, S.R.; Dudek, H.; Tao, X.; Masters, S.; Fu, H.; Gotoh, Y.; Greenberg, M.E. Akt phosphorylation of Bad couples survival signals to the cell-intrinsic death machinery. Cell 1997, 91, 231-241.

35. Brunet, A.; Bonni, A.; Zigmond, M.J.; Lin, M.Z.; Juo, P.; Hu, L.S.; Anderson, M.J.; Arden, K.C.; Blenis, J.; Greenberg, M.E. Akt promotes cell survival by phosphorylating and inhibiting a forkhead transcription factor. Cell 1999, 96, 857-868.

36. Mayo, M.W.; Baldwin. A.S. The transcription factor NF- $\kappa$ B: control of oncogenesis and cancer therapy resistance. Biochim. Biophys. Acta. 2000, 1470, M55-M62.

37. Hay, N.; Sonenberg, N. Upstream and downstream of mTOR. Gene Dev. 2006, 18, 1926-1945.

38. Shukla, S.; MacLennan, G.T.; Marengo, S.R.; Resnick, M.I.; Gupta, S. Constitutive activation of PI3K-Akt and NF-kB during prostate cancer progression in autochthonous transgenic mouse model. Prostate 2005, 64, 224-239.

39. Kaplan-Lefco, P.J.; Chen, T-M.; Ittman, M.M.; Barrios, R.J.; Ayala, G.E.; Huss, W.J.; Maddison, L.A.; Foster, B.A.; Greenberg, N.M. Pathobiology of autochthonous prostate cancer in a pre-clinical transgenic mouse model. Prostate 2003, 55, 219-237.

40. Gingrich, J.R.; Barrios, R.J.; Foster, B.A.; Greenberg, N.M. Pathologic progression of autochthonous prostate cancer in TRAMP model. Prostate Cancer Prostatic Dis. 1999, 2, 70-75.

(C) 2011 by the authors; licensee MDPI, Basel, Switzerland. This article is an open access article distributed under the terms and conditions of the Creative Commons Attribution license (http://creativecommons.org/licenses/by/3.0/). 